\title{
An acute ST-elevation myocardial infarction which went viral
}

\author{
Chui King Wong' ${ }^{1 *}$, Glen Chiang Hong $\operatorname{Tan}^{2}{ }^{\circledR}$, Mohd Johar Jaafar ${ }^{\circledR}$ \\ 'Department of Emergency Medicine, Faculty of Medicine, Universiti Kebangsaan Malaysia Medical Centre, Jalan \\ Yaacob Latif, 56000 Kuala Lumpur, Malaysia \\ 2Department of Emergency Medicine, Hospital Tengku Ampuan Rahimah, Jalan Langat, 41200 Klang, Selangor Darul \\ Ehsan, Malaysia
}

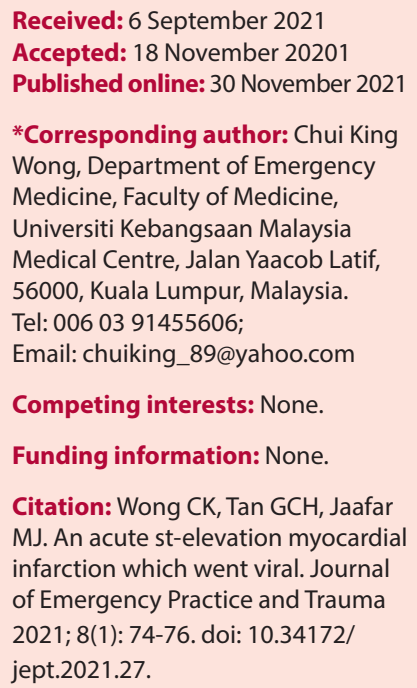

\begin{abstract}
Objective: ST-elevations in electrocardiogram (ECG) secondary to an acute myocarditis may mimic ST-elevation myocardial infarction (STEMI). It is vital to distinguish between the two entities to avoid inappropriate clinical management and complications.

Case Presentation: A previously well 19-year-old male presented with two episodes of central chest pain which were resolved spontaneously. His presentation was preceded by multiple episodes of vomiting, diarrhoea and abdominal pain. Physical examination was unremarkable except for a low-grade temperature of $37.7^{\circ} \mathrm{C}$. The first ECG revealed ST-segment elevations in anterior leads without reciprocal changes. Serial ECGs showed increasing ST elevations and his cardiac markers were significantly raised. As the initial clinical presentation was potentially an acute coronary syndrome, he was instinctively treated with anti-platelets. Fortunately, this patient was not given thrombolysis as there were clinical suspicions of an acute myocarditis due to his young age, presence of viral symptoms, and absence of cardiac risk factors. Subsequent cardiac MRI confirmed the diagnosis of an acute myocarditis.

Conclusion: An acute myocarditis is well known but less common presentation of viral infections. ST-segment elevations in ECG of any young patients with chest pain but without risk factors for acute coronary syndrome should always raise the suspicion of acute myocarditis especially in the presence of viral symptoms. Investigations such as cardiac magnetic resonance imaging (MRI) should be carried out emergently to distinguish both conditions.
\end{abstract}

Keywords: Myocarditis, ST Elevation Myocardial Infarction, Viral infections

\section{Introduction}

Myocarditis, or inflamed myocardium, has various possible etiologies such as infections, cardiac toxins, hypersensitivity reactions, systemic disorders and even radiation exposure. Amongst all infectious etiologies, viral infections are the commonest culprit for myocarditis. Myocarditis has a broad spectrum of clinical manifestations and it may even mimic an acute STelevation myocardial infarction (STEMI). Any patients presenting with a typical chest pain associated with ST elevation on their electrocardiogram (ECG) with elevated cardiac biomarkers should always make doctors suspicious of STEMI because it requires timely intervention (1). However, it is important to note that a misdiagnosis of STEMI in patients suffering from myocarditis will result in unnecessary fibrinolysis or even percutaneous coronary intervention with potentially detrimental complications. Since the treatment options for STEMI are significantly different from that of an acute myocarditis, hence, an accurate diagnosis is crucial.

\section{Case Presentation}

A healthy 19-year-old male presented to the non-critical area of the emergency department with complaints of vomiting, diarrhoea and abdominal pain for the past two days. He also had two episodes of central chest pain at rest that was resolved spontaneously over the same duration. However, the pain was not associated with autonomic or failure symptoms. Upon arrival, his temperature was $37.7^{\circ} \mathrm{C}$ with blood pressure of $104 / 63 \mathrm{~mm} \mathrm{Hg}$ and pulse rate of 79 beats/min. His cardiovascular examination was normal without any evidence of murmur, pericardial rub or gallop rhythm. Other systemic examinations were unremarkable. His initial ECG showed ST-segment elevations from leads V2 - V6 without reciprocal changes (Figure 1). Serial ECG revealed increasing ST elevations 


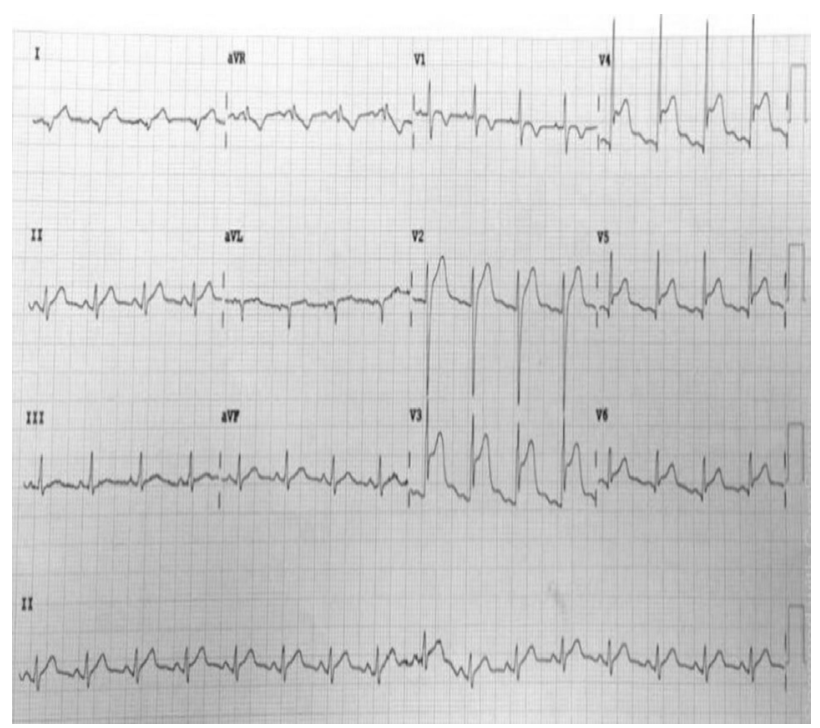

Figure 1. Initial ECG shows ST elevations from leads V2-V6.

(Figure 2). His cardiac biomarkers were significantly raised with serum CK-MB of $76 \mathrm{U} / \mathrm{L}$ and high sensitivity troponin $\mathrm{T}$ of $1.585 \mu \mathrm{g} / \mathrm{L}$. Total white cell counts were also raised at $18.3 \times 10^{9} / \mathrm{L}$. His chest radiograph was unremarkable. Also, echocardiography showed mild hypokinesia of the anterolateral walls with reduced ejection fraction of $30 \%$ to $40 \%$. As the initial clinical picture painted a possible acute coronary syndrome, he was empirically loaded with double anti-platelets without thrombolytic therapy or anticoagulants. The working diagnosis was that of an acute myocarditis due to his young age and absence of risk factors for acute coronary syndrome. He was later transferred to a heart center for further management but he remained asymptomatic throughout admission. In-patient cardiac magnetic resonance imaging (MRI) with late gadolinium enhancement revealed sub-epicardial hyperenhancement from the basal to apical lateral wall, basal to apical

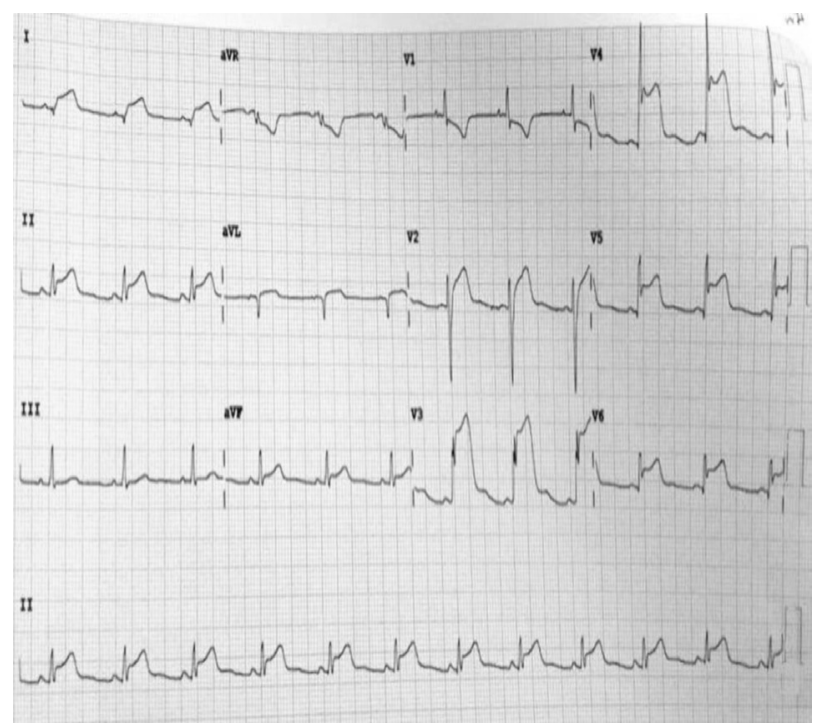

Figure 2. Repeated ECG shows worsening ST elevations from leads V2 -V6 without reciprocal changes. inferior wall that extends to mid anterior wall and also a mid-wall fibrosis at mid septal region (Figure 3). The T2-weighted triple-inversion recovery showed increased signal intensity with edema which is consistent with the diagnosis of an acute myocarditis. After the diagnosis of acute myocarditis was established, his anti-platelets were stopped and we started oral perindopril for him. He was discharged after a week of hospital admission with an outpatient follow-up. He appeared well during follow-up with normal echocardiography findings after four months.

\section{Discussion}

The incidence of viral myocarditis is reported to be around the range of 10 to 22 cases per 100000 individuals annually (2). Myocarditis may be present in $1 \%-5 \%$ of all patients who are tested positive for acute viral infections. The viruses commonly linked to acute myocarditis are the coxsackievirus, adenovirus, parvovirus B19, influenza, cytomegalovirus, hepatitis $\mathrm{C}$ virus, human immunodeficiency virus, herpes simplex virus and Epstein-Barr viruses.

The clinical presentation of acute myocarditis is so variable that it may be completely symptomless or it may present as cardiogenic shocks and even sudden cardiac arrests at the other extreme. It poses a diagnostic challenge to clinicians because the clinical manifestation may mimic an acute myocardial infarction. In this case, although the young man had recurrent resting chest pain with signs of myocardial injury like ST elevations and elevated cardiac markers, the history of preceding acute gastroenteritis in a young patient without coronary risk factors favoured the diagnosis of a myocarditis.

The pathogenesis of viral myocarditis can be divided into three phases; the phase of direct myocardial

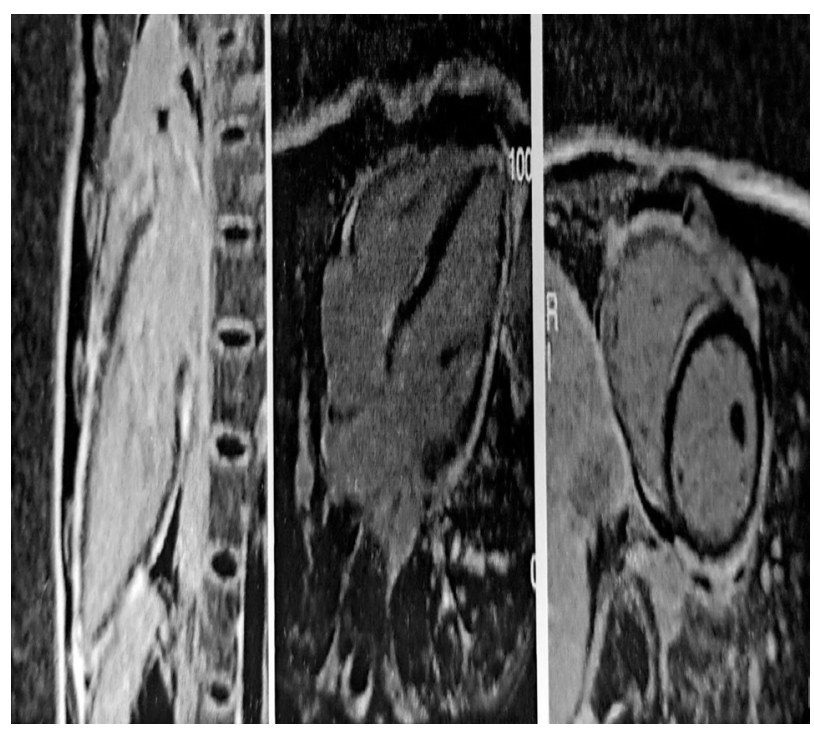

Figure 3. Cardiac MRI: late gadolinium enhancement imaging shows sub-epicardial hyperenhancement from the basal to apical lateral wall and basal to apical inferior wall that extends to mid anterior wall. There is also mid-wall fibrosis at mid septal region. 
damage, followed by dysregulated immune response and subsequently extensive myocardial damage with dilated cardiomyopathy. Virus invasion into the myocardial tissue during the first phase leads to focal myocardial injury that manifests as ST elevation on ECG and raised cardiac markers, mimicking a STEMI. This teenager was in the first phase of the disease upon arrival to our emergency department with ST elevations on his ECG and raised cardiac markers. During the second phase, an autoimmune response may occur due to the molecular mimicry between the epitopes of the viral and cardiac myocytes. Patients usually progress into heart failure at this stage. The inflammation can then progress to extensive myocardial damage and fibrosis leading to dilated cardiomyopathy, the final stage of the disease $(3,4)$.

There are different diagnostic modalities available for myocarditis. The gold standard for the diagnosis of myocarditis is through endomyocardial biopsy, but the procedure is invasive and not readily available in all centers. The sensitivity of endomyocardial biopsy to accurately diagnose myocarditis is up to $75 \%$ (5). In patients with typical features of acute coronary syndrome and significant coronary risk factors, a selective angiography or CT coronary angiography is recommended to rule out thrombosis. Cardiac MRI is another imaging modality that is increasingly being utilized by clinicians to diagnose myocarditis. This non-invasive test has the ability to determine the extent of the inflammation and also help to delineate the normal from the inflamed tissues (6). This allows Cardiac MRI to be used as a guide for endomyocardial biopsy to increase the sensitivity and the specificity of the test to detect myocarditis. However, it is still not readily available in most centers, hence; relying purely on it to differentiate myocarditis from an acute coronary syndrome during an acute presentation may significantly delay time-critical treatment. In summary, the diagnosis of an acute viral myocarditis should still be made based on the clinical suspicion from clinical history and also serial ECG in the acute setting to prevent mistreatment.

\section{Conclusion}

Physicians should exercise caution in treating patients from the atypical group for acute myocardial infarction. Young patients without any traditional coronary risk factors presenting with infective symptoms should always raise the suspicion of myocarditis. Investigations such as coronary angiography and Cardiac MRI are recommended to distinguish between these two clinical entities to guide clinical management.

\section{Authors' contributions}

WCK contributed to the clinical management of the patient, writing of the manuscript, approval of the final draft of the manuscript and corresponding author. MJJ and GTCH contributed to the support of the literature, revision and approval of the final draft of the manuscript.

\section{Ethical issues}

Written informed consent was obtained from the patient for his anonymized information to be published.

\section{References}

1. Ibanez B, James S, Agewall S, Antunes MJ, BucciarelliDucci C, Bueno H, et al. 2017 ESC Guidelines for the management of acute myocardial infarction in patients presenting with ST-segment elevation: the Task Force for the management of acute myocardial infarction in patients presenting with ST-segment elevation of the European Society of Cardiology (ESC). Eur Heart J 2018; 39(2): 11977. doi: 10.1093/eurheartj/ehx393.

2. Vos T, Barber RM, Bell B, Bertozzi-Villa A, Biryukov S, Bolliger I, et al. Global, regional, and national incidence, prevalence, and years lived with disability for 301 acute and chronic diseases and injuries in 188 countries, 1990-2013: a systematic analysis for the Global Burden of Disease Study 2013. Lancet 2015; 386(9995): 743-800. doi: 10.1016/s01406736(15)60692-4.

3. Li YD, Hsiao FT, Lai CP, Chen CW. Acute viral myocarditis mimicking ST elevation myocardial infarction: manifestation on cardiac magnetic resonance. Acta Cardiol Sin 2010; 26: 44-7.

4. Zhang T, Miao W, Wang S, Wei M, Su G, Li Z. Acute myocarditis mimicking ST-elevation myocardial infarction: a case report and review of the literature. Exp Ther Med 2015; 10(2): 459-64. doi: 10.3892/etm.2015.2576.

5. Van Linthout S, Tschöpe C. Viral myocarditis: a prime example for endomyocardial biopsy-guided diagnosis and therapy. Curr Opin Cardiol 2018; 33(3): 325-33. doi: 10.1097/hco.0000000000000515.

6. Ang KP, Quek ZQ, Lee CY, Lu HT. Acute myocarditis mimicking ST-elevation myocardial infarction: a diagnostic challenge for frontline clinicians. Med J Malaysia 2019; 74(6): 561-3. 